\title{
Hybrid Particle Swarm Optimization of Electricity Market Participation Portfolio
}

\author{
Ricardo Faia ${ }^{1}$, Tiago Pinto ${ }^{1,2}$, Zita Vale ${ }^{1}$, Juan Manuel Corchado ${ }^{2}$ \\ ${ }^{1}$ GECAD - Research Group on Intelligent Engineering and Computing for Advanced Innovation and Development \\ Polytechnic of Porto (ISEP/IPP), Porto, Portugal \\ $\{$ rfmfa, tmcfp, zav $\} @$ isep.ipp.pt \\ ${ }^{2}$ BISITE Research Centre, University of Salamanca (USAL), Calle Espejo, 12, 37007 Salamanca, Spain \\ \{tpinto, corchado\}@usal.es
}

\begin{abstract}
This paper proposes a novel hybrid particle swarm optimization methodology to solve the problem of optimal participation in multiple electricity markets. The decision time is usually very important when planning the participation in electricity markets. This environment is characterized by the time available to take action, since different electricity markets have specific rules, which requires participants to be able to adapt and plan their decisions in a short time. Using metaheuristic optimization, participants' time problems can be resolved, because these methods enable problems to be solved in a short time and with good results. This paper proposes a hybrid resolution method, which is based on the particle swarm optimization metaheuristic. An exact mathematical method, which solves a simplified, linearized, version of the problem, is used to generate the initial solution for the metaheuristic approach, with the objective of improving the quality of results without representing a significant increase of the execution time.
\end{abstract}

Index Terms - Artificial intelligence, electricity markets, hybrid resolution methods, portfolio optimization, metaheuristic optimization

\section{INTRODUCTION}

Hybrid optimization algorithms are characterized by combining different types of optimization methods. Different combinations of optimizations methods have been used in the scientific community: exact methods combined with other exact methods, exact methods combined with metaheuristics, and combinations of different metaheuristics [1]. Metaheuristic does not have a standard definition, however, a widely accepted characterization is provided by Osman and Laporte [2]: " $A$ metaheuristic is formally defined as an iterative generation process which guides a subordinate heuristic by combining intelligently different concepts for exploring and exploiting the search space, learning strategies are used to structure information in order to find efficiently near-optimal solutions."

Metaheuristic approaches are especially useful for reaching good solutions for heavy computational problems in fast execution times. This is especially relevant when solving realworld problems, in which the decision time is a relevant decision factor. The participation in electricity markets is one of these problems due to the significant changes during the last decades [3]. Before the liberalization of electricity markets, the

The present work was done and funded in the scope of the following projects: H2020 ADAPT Project (Marie Sklodowska-Curie grant agreement No 703689), DREAM-GO Project (Marie Sklodowska-Curie grant agreement No 641794) and AVIGAE Project (P2020 - 3401). system operator considered demand to be fixed and scheduled operation plans based on generation resources. This made electricity negotiations highly restricted, mostly due to the fact that much of the produced energy came from fully controllable generation sources.

The recent energy policy has favored a massive introduction of renewable energy sources on electricity markets, which has greatly impacted their penetration in power systems [4]. The intermittent nature of renewable energy had a very large impact on the way the negotiations operated, since the energy supply directly influences the market prices. At the market level the competition has increased, bringing a greater number of sellers to participate [5]. This also leads to the emergence of aggregators to represent (and even manage) groups of small player [6], in order to increase their impact on the market. A market dimension is also being introduced into retail markets, in order to motivate consumers to change their passive attitudes. The enlargement of metering infrastructure is now creating the means to enable consumers to participate in competitive retail markets by overcoming the lack of infrastructure that enables sending market signals to and from consumers [7].

With the increase in competitiveness in electricity markets [8], caused by the increase of energy producers (in particular renewables), there is an increasing need for tools that can provide support to electricity market participants. Multi-Agent Simulator of Competitive Electricity Markets (MASCEM) [9] is integrated with a decision support system that aims at providing market players with suitable suggestions on which actions should be performed at each time and in different contexts of negotiation. This system is Adaptive Decision Support for Electricity Markets Negotiations (AiD-EM). AiDEM is itself composed by several distinct decision support systems, directed to the negotiation in different EM types; e.g. Adaptive Learning Strategic Bidding System (ALBidS) [10].

After this introduction, section II presents an overview of the related work the field, and section III presents the mathematical formulation of the addressed problem. Section IV details the proposed hybrid model based on particle swarm optimization methodology. Section V presents the results of the case study, and finally, section VI presents the most relevant conclusions of this work. 


\section{RELATED WORK}

Markowitz proposed the Markowitz Portfolio Selection Theory in 1952 [11]. This theory enables combining assets in such a way that the resulting portfolio is characterized by a higher return to risk ratio, when compared to that provided by every single asset by itself, an effect known as diversification; i.e. the more diversified the portfolio, the lower risk level.

The concepts of portfolio optimization and diversification are instrumental in the development and understanding of financial markets and financial decision making. However, in the last years, the theory of portfolios has been applied in the electricity markets area, with the purpose of supporting the decision making [12]. Using the portfolio optimization results, market participants can obtain a better market participation, by obtaining a larger profit / lower cost from their participation.

In [10], the authors propose a portfolio optimization for multiple electricity market participation. In this methodology, the players can sell and buy electricity in several markets as well as selling the produced energy. A risk management model is also considered, but it considers that risk is originating from the prices forecasting. The prices forecasting is made through an artificial neural network (ANN) [13] and three different values for each case are presented. These three values are considered as the risk levels. The risk is associated with the expected price because with the simulations ANN arrives to different values for the same case. The maximum, minimum and average values for all simulations are calculated, in order to feed the optimization problem. A Particle Swarm Optimization (PSO) [14] variant is used to solve the optimization problem.

In [15], the authors propose a methodology for risk analysis and portfolio optimization of power assets with hydro, wind and solar power. In case study the authors considering the Regulated Contacting Environment and the Mechanism for Reallocation of Energy Brazil.

When the execution time for reaching a solution for these problems is a relevant decision factor, metaheuristic optimization approaches are often applied. Metaheuristics can be based on [16]: single-solution, if they use a single starting point (e.g. local search, simulated annealing, iterated local search and tabu search) or population-based if a population search points is used (e.g. particle swarm, evolutionary algorithms, colony based optimization). Many of these approached are inspired by natural processes (e.g. evolutionary algorithms from biology or simulated annealing from physics).

In metaheuristics search there two ways to characterize the research: exploration of the search space (diversification) and exploitation of the best fond solution (intensification). Exploration means the diversification of the search to different regions in the search space for a better sampling of the solution space. In the other hand, exploitation means the intensification of the search around some the good quality solutions in other to find an improved solution. A balance between two contradictory objectives must therefore be guaranteed [17]. When applying these strategies to the solution of any optimization problem, the main concern is to determine the algorithm capability for finding the global optimum. The desirable feature of an effective optimization method is a high successes probability for finding the global solution ate the expenses the lower computational efforts. Theoretically, it is important to remark that stochastic methods (metaheuristics) need an infinite number of objective function evaluations to guarantee the convergence of the global optimum. This number is determined by the parameters employed for controlling the search process (exploitation and exploration) and the termination criterion [18]. There are many stopping criterion used in stochastic optimization methods: they are based on the measurement of the relative error to the know value of the global optimum, the improvement of the value of objective function for a certain number of iterations or functions evaluations, or a maximum allowable numerical effort that is defined in terms of the number of algorithm iterations or objective function evaluations.

In summary, the optimal solution cannot be guarantee when using a metaheuristic, but a reasonable good solution is obtained without having to explore the whole solutions space, and consequently in a much shorter time, when compared to resolution by exact methods. There are different metaheuristics that can be applied, these vary depending on the search heuristic method chosen to guide the search. Thus, each metaheuristic can present different results. In real world applications, the main interest is in obtaining a good solution in a reasonable amount of time. Therefore, metaheuristic methods are highly appreciated as efficient means for dealing with real-world applications [19].

\section{MATEMATICAL FORMULATION}

The formulation presented in (1) is used to represent the optimization problem, as proposed in [20] . In (1) $d$ represents the weekday, $N$ day represent the number of days, $p$ represents the negotiation period, Nper represent the number of negotiation periods, Asell $_{M}$ and $A b u y_{S}$ are boolean variables, indicating if this player can enter in negotiation in each market type, $M$ represents the referred market, NumM represents the number of markets, $S$ represents a session of the balancing market, and NumS represents the number of sessions. Variables $p s_{M, d, p}$ and $p s_{S, d, p}$ represent the expected (forecasted) prices of selling and buying electricity in each session of each market type, in each period of each day. The outputs are $S_{\text {pow }}$ representing the amount of power to sell in market $M$ and $B p o w_{S}$ representing the amount of power to buy in session $S$.

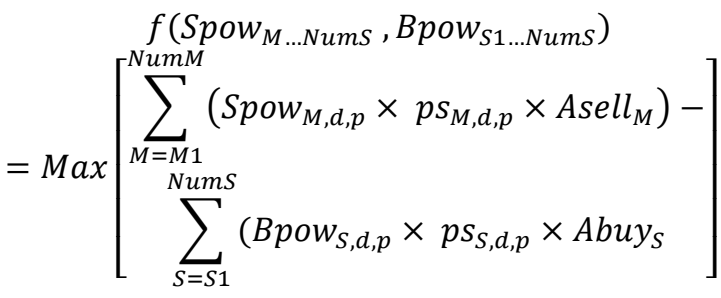

$$
\begin{aligned}
& \forall d \in N d a y, \forall p \in N p e r, \text { sell }_{M} \in\{0,1\}, \text { Abuy } \\
& \in\{0,1\} \\
& p s_{M, d, p}=\operatorname{Value}\left(\operatorname{Spow}_{M}\right)_{M, d, p} \\
& p s_{S, d, p}=\operatorname{Value}\left(\text { Bpow }_{S}\right)_{S, d, p}
\end{aligned}
$$


The formulation considers the expected production of a market player for each period of each day. The price value of electricity in some markets depends on the power amount to trade. With the application of a clustering mechanism it is possible to apply a fuzzy approach to estimate the expected prices depending on the negotiated amount. Equation (2) defines this condition.

$$
\begin{aligned}
\operatorname{Value} & \left(\operatorname{Spow}_{M} \text { or Bpow }\right)_{d, p, M, S} \\
& =\operatorname{Data}\left(\operatorname{fuzzy}_{(\mathrm{pow}))_{d, p, M, S}}\right.
\end{aligned}
$$

Equation (3) represents the main constraint to be applied in this type of problems, and imposes that the total power that can be sold in the set of all markets is never higher than the total expect production (TEP) of the player, plus the total of purchased power [20]. Restrictions (4), (5) and (6) refer to the type of generation of the supported player.

$$
\begin{gathered}
\sum_{M=M 1}^{N u m M} \text { Spow }_{M} \leq T E P+\sum_{S=S 1}^{\text {Nums }} \text { Bpow }_{S} \\
T E P=\sum_{\in} \text { Energy }_{\text {prod }}, \text { Energy }_{\text {prod }} \\
\in\left\{\text { Renew }_{\text {prod }}, \text { Therm }_{\text {prod }}\right\} \\
0 \leq \text { Renew }_{\text {prod }} \leq \text { Max }_{\text {prod }} \\
\text { Min }_{\text {prod }} \leq \text { Therm }_{\text {prod }} \leq \text { Max }_{\text {prod }}, \text { if } \text { Therm } \\
>0
\end{gathered}
$$

From the presented restrictions and considerations one can see that the energy produced comes from renewable sources and non-renewable sources (thermoelectric). If the player is a producer of thermoelectric power, the production has to either be null or set at a minimum value, since it is not feasible for the production plant to work under a technical operation limit. If the player is a producer of renewable energy, the only restriction is the maximum production capacity.

\section{PROPOSED HYBRID APPROACH}

The methodology proposed in this work is created to solve the portfolio optimization problem. In this case two different methods are used, namely an exact resolution method and a stochastic resolution method (PSO). As it is possible to observe by Fig. 1, a simplified version of the problem optimization is done by using the exact method, using the Cplex solver to solve the Mixed Integer Linear Programing (MILP) problem. The solution that is achieved for the simplified version of the problem is then used as initial solution for the approximate method (PSO) to be executed.

All the metaheuristic optimization methods require an initial solution to start the optimization process (which is often randomly generated). The role of Cplex is to provide the initial solution for PSO to initialize the search. Usually the resolution of MILP problems, as indicated in the Fig. 1, can take along execution time, depending on the problem in hand, However, to circumvent this problem the solutions are only restricted to integers and the resolution time is quite acceptable (the comparison can be consulted in the case study presented in section $\mathrm{V}$ ).

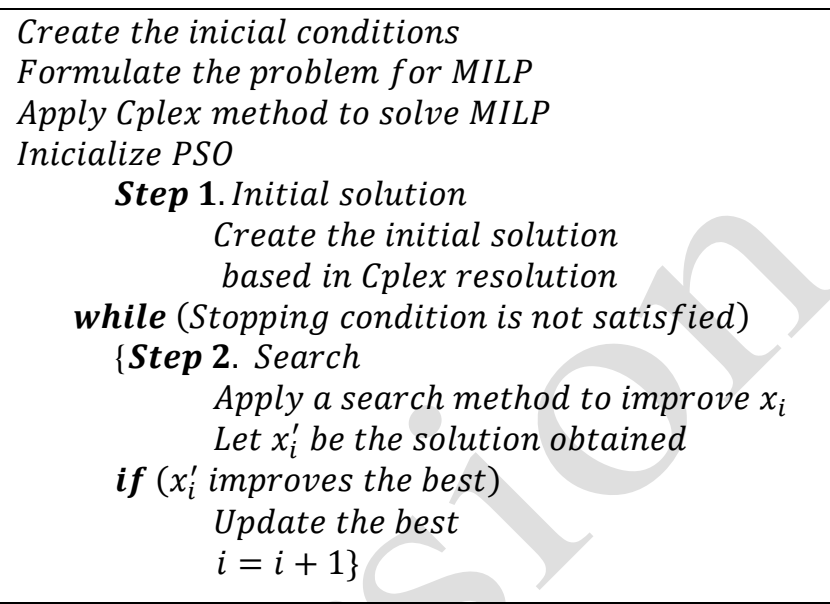

Fig. 1. Hybrid methodology pseudo code

As can be followed by Fig. 1, after performing the optimization by Cplex, the iterative search process, the PSO, is started. In the first step the initial solution is created so that the PSO method starts its search. Different variants are experimented and compared in the case study of section $\mathrm{V}$ for the creation of the initial solution, but all based on the solution from Cplex.

The PSO algorithm does not guarantee the global optimal solution, generally the search is stopped when the stopping criteria is reached. At each iteration PSO applies equation (7) and equation (8). During the iterations of the algorithm each particle of PSO moves in the space with a velocity that is dynamically adjusted (different in each iteration). The velocity determines particles' positions according to their own and their neighboring-particles experiences, thus moving two points in each iteration: (i) the best position found so far by itself, called Pbest; and (ii) the best position of all neighbor particles, called Gbest [14].

$$
\begin{gathered}
v_{i d}^{k+1}=w \cdot v_{i d}^{k}+c_{1} \cdot r_{1}^{k} \cdot\left(\text { Pbest }_{i d}^{k}-x_{i d}^{k}\right) \\
+c_{2} \cdot r_{2}^{k} \cdot\left(\text { Gbest }_{i d}^{k}-x_{i d}^{k}\right) \\
x_{i d}^{k+1}=x_{i d}^{k}+v_{i d}^{k+1}
\end{gathered}
$$

Where,

- $v_{i d}^{k}$ - velocity of particle $\mathrm{i}$, parameter $\mathrm{d}$ and iteration $\mathrm{k}$,

- $x_{i d}^{k}$ - position of particle $\mathrm{i}$, parameter $\mathrm{d}$ and iteration $\mathrm{k}$,

- $k$-iteration,

- Pbest-personal best,

- Gbest-global best,

- $\quad w$ - inertia term,

- $c_{1}$ - local attraction term.

- $c_{2}$ - global attraction term.

- $r_{1}, r_{2}$ - random numbers between $[0,1]$. 
Afterwards, equation (7) and (8) are applied to find new positions for each particle, and the fitness is calculated by using the objective function, equation (1). The next step is to update the best individual positions (Pbest $t_{i}$ ) if the current position of a particle is the best found so far by that particle. Pbest is also compared to the best global position (Gbest). If Pbest $t_{i}$ is better than Gbest, Gbest is also updated to the new position.

\section{CASE STUdy}

This section presents the case study that illustrates the application of the proposal methodology. As has been previously started, the PSO algorithm starts with an initial solution based on the Cplex resolution. In this scenario, five different markets type have been considered, the considered markets are the day-ahead spot market, negotiations by means bilateral contacts, the balancing or intra-day market, and a local market, at the Smart-Grid (SG) level.

The balancing market is divided into different sessions. In the day-ahead spot market the player (acting as seller) is only allowed to sell electricity, while in the other market types the player can either buy or sell depending on the expected prices. Limits have also been imposed on the possible amount of negotiation in each market. In this case, it is only possible to buy up to $10 \mathrm{MW}$ in each market in each period of negotiation, which makes a total of $40 \mathrm{MW}$ purchased. It is possible to sell power on any market, and it can be transacted as a whole or in installments. The player has $10 \mathrm{MW}$ of own production (TEP) for sale.

Table 1 shows the initial solution achieved by the Cplex method. For this resolution the Tomlab toolbox of Matlab has been used. Cplex 2 represents the version of Cplex resolution used to generate the initial solution. In this case, the variables that constitute the solution are only positive integer values; this particularity greatly simplifies the method, enabling it to solve the problem in a short execution time. In Table 2 Cplex 1 represents the complete version of Cplex resolution (when the variables of the solution can be positive rational numbers), which leads to high execution times.

Table 1. Cplex result for initial solution

\begin{tabular}{ccccccc}
\hline \hline \multicolumn{2}{c}{ Method } & Spot & Bilateral & Balancing 1 & Balancing 2 & Smart Grid \\
\hline \multirow{2}{*}{ Cplex2 } & Sell & 14 & 12 & 0 & 0 & 9 \\
& Buy & 0 & 5 & 10 & 10 & 0 \\
\hline \hline
\end{tabular}

Table 1 presents the optimized variables, assuming only integer values; this configuration of the solution allows to obtain an objective function value present in the Table 2 . In the Spot market, $14 \mathrm{MW}$ are sold, $12 \mathrm{MW}$ by means of bilateral contracts and $9 \mathrm{MW}$ in $\mathrm{SG}$, in the variables that represent the purchase can see that in the balance markets the maximum quantity (10 MW) is bought, and it will also buy $5 \mathrm{MW}$ to bilateral markets.

In the bilateral contracts, as can be observed in the variables, the two actions are performed, buying and selling. This is possible due to the fact that in this market the quantity of electricity traded influences the price of it. In the SG market, there is also this possibility but the resolution by this method does not present this possibility.
After obtaining the initial solution, it is tested as input for different versions of the PSO. These versions differ in the construction of the initial solution. Initially it is considered that only one particle receives the solution of Cplex2, and thus the other particles will have a random solution, this version is called "Hybrid PSO".

In order to understand the influence of the initial solution in the PSO research, 4 more versions were created. In these versions all the particles receive solutions built from solution of the Cplex2. In the solution construction phase is used the normal distribution, since, as explained in section IV, since the problem has ten variables, each variable must have a value for PSO to start the search. Fig. 2 presents three representations of normal distribution, varying the Standard Deviation (STD), for the first variable: sell in spot market,.

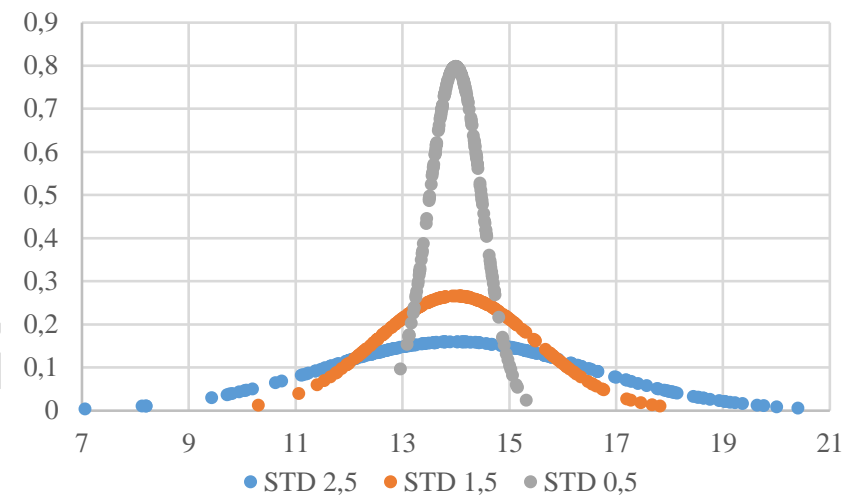

Fig. 2. Normal distribution for mean $=14$

The normal distribution is characterized by the mean and the STD. In PSO, the initial solution requires one solution for each variable, so 1 particle has ten different variables. The representation in Fig. 2 demonstrates the influence of the STD in the creation of the initial solution, since the average of the distribution was used the value of the variable of sale in the spot market optimized by the Cplex 2 present in Table 1 , this process is repeated for all variables and for each particle.

From the analysis of the Fig. 2 one can verify that the bigger the STD the greater the dispersion of the distribution, and the possible range values for each variable increase. E.g. if $\mathrm{STD}=0.5$ there is higher probability of the value created to be closer to the value of the mean than if STD $=2.5$. In this case, the STD value allows the creation of different initial solutions to the problem using the variables that resulted from the Cplex2 resolution.

Table 2 presents the objective function results for all methods. The STD $=0.5$ version represents the hybrid PSO that starts its search with a solution created from a normal distribution with mean corresponding to the deterministic resolution value (Cplex2, Table 1) and with $\mathrm{STD}=0.5$. The versions of $\mathrm{STD}=1.5$ and $\mathrm{STD}=2.5$, is exactly the same as version $\mathrm{STD}=0.5$, but the value of the standard deviation is respectively 1.5 and 2.5 . 
Table 2. Objective function results $(€)$

\begin{tabular}{ccccc}
\hline \hline Method & Min & Mean & Max & STD \\
\hline Cplex1 & & & 2000,64557539 & \\
Hybrid PSO* $^{*}$ & 2000,36 & 2000,63 & 2000,64557501 & 0,033 \\
STD 2,5* & 1978,81 & 1999,96 & 2000,64557488 & 1,605 \\
STD 1,5* & 1980,38 & 2000,28 & 2000,64557444 & 1,552 \\
STD 2,5 (>=0) & 1999,82 & 2000,54 & 2000,64557339 & 0,104 \\
STD 0,5* & 1990,82 & 2000,06 & 2000,64557292 & 1,155 \\
PSO & 1135,39 & 1809,85 & 2000,64555238 & 150 \\
Cplex2 & & & 1998,84249084 & \\
\hline \hline
\end{tabular}

${ }^{*}$ Cplex2 plus PSO

The version $\mathrm{STD}=2.5(>=0)$, represents the hybrid method when all PSO particles start with a solution generated by a normal distribution with $\mathrm{STD}=2.5$, and all variables in all solutions have some positive values. The Cplex1 and Cplex2 only have a maximum number for objective function because these are deterministic methods and not population based. The other resolutions have different measurements because for each method 1000 simulations are executed. Table 2 shows the values of objective function for all implemented methods. As expected, the deterministic resolution (Cplex1) reached the best objective function value, followed by the Hybrid PSO where only one of the particles starts with the initial solution of Cplex2. Next, the hybrid versions appear, in which the initial solution of all the particles was created using the normal distribution. Finally appears the PSO where the initial solution was created by random values and finally appears the Cplex 2 .

As one can see, the difference between Cplex 1 and PSO in the objective function value for the maximum is 0.000023 , it is a residual value. In the average parameter, there is already a larger difference, but the hybrid PSO has a value very close to Cplex1 (reference result) with a difference of 0.0155 . The greater difference in results is observed in the STD of the methods including the PSO. Fig. 4 shows a representation of the convergence process of the different versions of the PSO, by showing the convergence in all the 1000 executions. This enables assessing how the STD stands for in the solution search using random methods.

In Fig. 4, six different representations are presented, which refer to the results of the PSO algorithms. Since 1000 runs were performed for each, each algorithm obtains 1000 different results, and each line in the figure represents the evolution of the solution throughout the iterations. As one can see, the images of the Fig. 4 do not have the scales in the same magnitude, which may make it difficult to observe, but with the scales all the same, it was not possible to have the notion of what really happens in the convergence process. In Fig. 4 a) is represented the standard PSO, which has an STD of 150, as can be seen by Table 2. One can observe that this resolution presents the simulations with very different final results hence the existence of the large STD. Also, in Fig. 4 is represented the Hybrid PSO method, which results, from Table 2, in the lowest value of STD. Then the methods with initial solution based on the normal distributions follow in terms of STD. Within these we can see that the STD of the 1000 simulations decreases depending on the STD of the normal distribution applied for the creation of the initial solution. It is important to mention that the method where only one of the particles starts with the initial solution obtained through Cplex 2 presents a better performance in terms of STD than the versions where all the particles receive a solution containing information from the solution of Cplex2.

Table 3 shows the comparison of execution times between all the considered methods. The "Total mean values" column represents the mean value of execution times. As noted, Cplex1 and Cplex 2 only have one value - exact solution, which means that it was only executed once. In the other cases, the values refer to the 1000 simulations.

Table 3.Time results for all methods in seconds

\begin{tabular}{|c|c|c|c|c|}
\hline Method & Mean & Max & $\begin{array}{l}\text { Total for all } \\
\text { runs }\end{array}$ & $\begin{array}{l}\text { Total mean } \\
\text { values }\end{array}$ \\
\hline Cplex1 & \multicolumn{3}{|c|}{43710,6809} & - \\
\hline Hybrid PSO & 0,0757 & 0,1613 & 75,7115 & 49,2461 \\
\hline STD 2,5 & 0,0759 & 0,1329 & 75,8515 & 49,2463 \\
\hline STD 1,5 & 0,0735 & 0,1191 & 73,4541 & 49,2439 \\
\hline $\begin{array}{c}\text { STD 2,5 } \\
(>=0)\end{array}$ & 407 & 0,1902 & 140,6565 & 49,3111 \\
\hline STD 0,5 & 0,0730 & 0,1234 & 72,9606 & 49,2434 \\
\hline PSO & 0,0613 & 0,1215 & 61,2705 & 22,6457 \\
\hline Cplex2 & \multicolumn{3}{|c|}{26,5859698} & - \\
\hline
\end{tabular}

By the Table 3 it is possible to verify that PSO presents the smaller value of execution time. As expected, the Cplex1 presents a high value but on the other hand guarantees the maximum value for the objective function. In this case the hybrid PSO methods and all other versions of the normal distribution have very similar values. It is worth noting that STD $2.5(>=0)$ takes twice the STD 2.5 due to the fact that the solutions are corrected to positive values.

In the column of "Total for all runs" the total value of the 1000 simulations is shown, which is proportional to the average value. In the last column, the total average value is displayed with all steps, from the data loads creation of the initial solution (Cplex2 time). The average value of the whole process is about 50 seconds for the hybrid PSO method, which is much smaller when compared to the value of Cplex1; however, the objective function value is very close. Fig. 3 shows the results for the number of iterations. In the bar graph the average value for each method is shown.

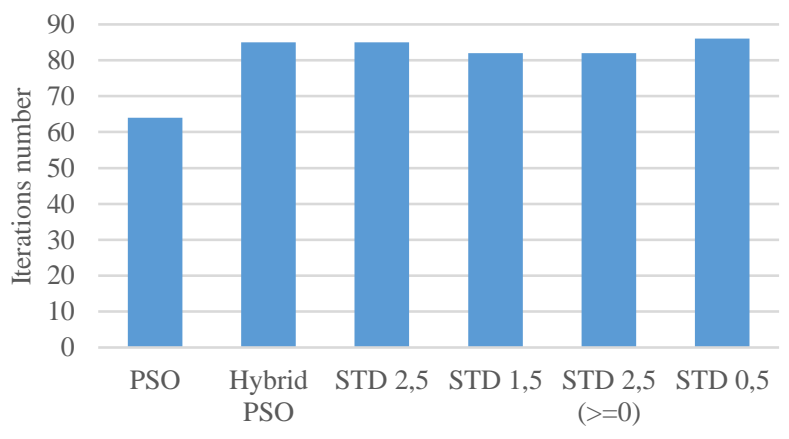

Fig. 3. Mean iteration results 
a)

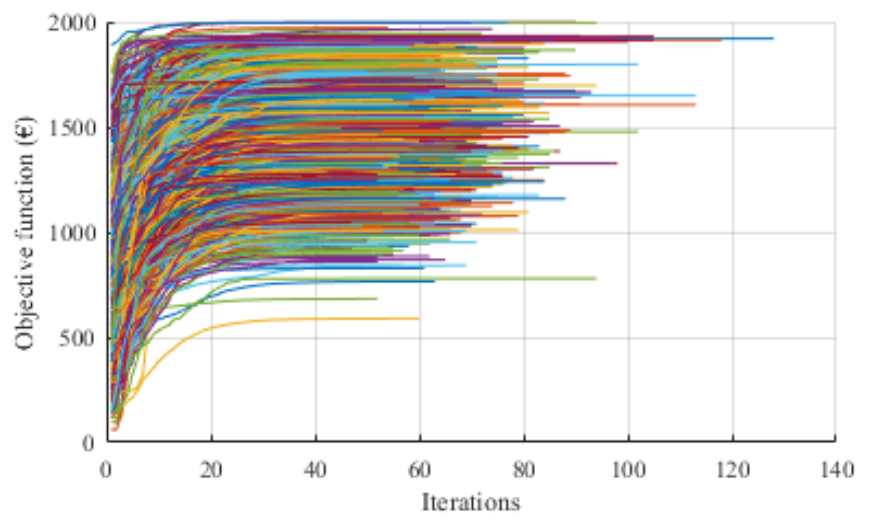

c)

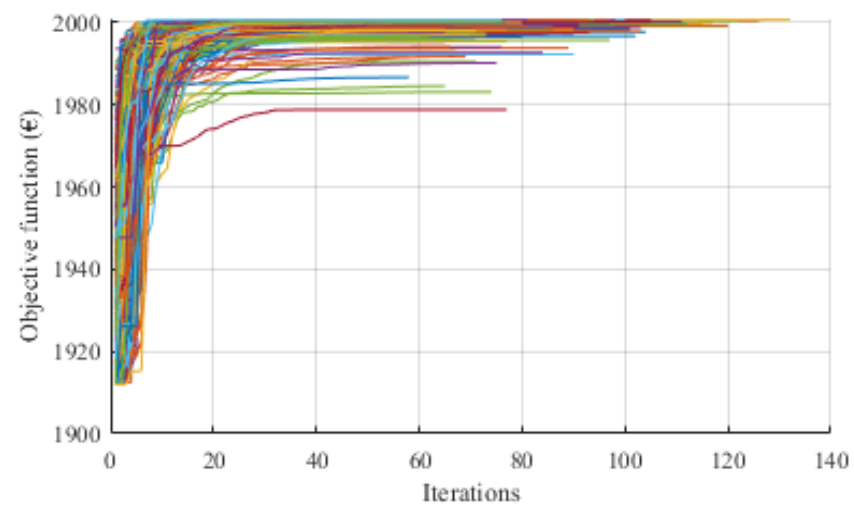

e)

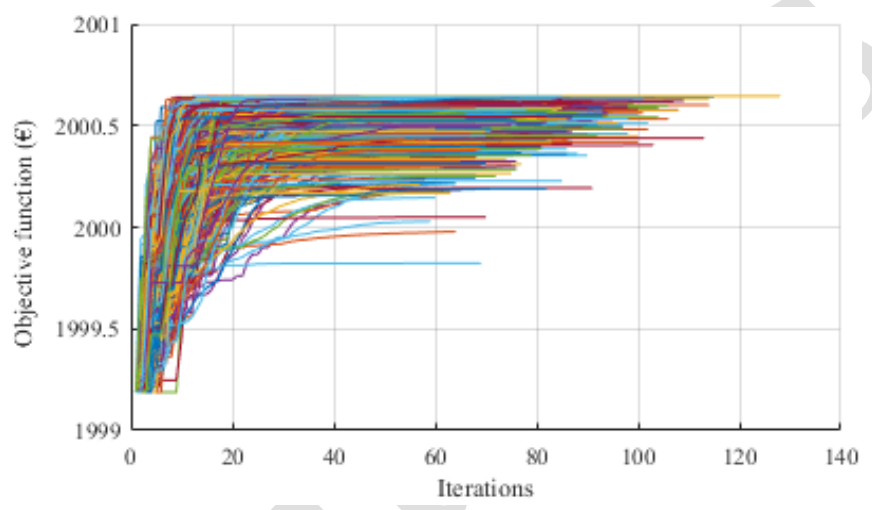

Fig. 4. Algorithms performance, a). PSO, b). Hybrid-PSO, c)

As can be observed by Fig. 3, the average number of iterations in the methods where the initial solution contains information of the resolution of Cplex 2 is between 80 and 90 iterations and in the PSO with random solution is about 63 . With the inclusion of the initial solution the average value of the iterations increases, this fact can be explained because in the PSO since the search has to start with random solution and often far away from the optimum, the search tends to fall in local points and the method does not have ability to get out of there, often converging to a bad solution.

In Fig. 5 is represented the value of the different variables in the different resolution methods. In the yy axis, the negative values represent the electricity purchases and the amount electricity sold is represented by positive values. In this case as can be seen from the caption of the Fig. 5 the 5 different b)

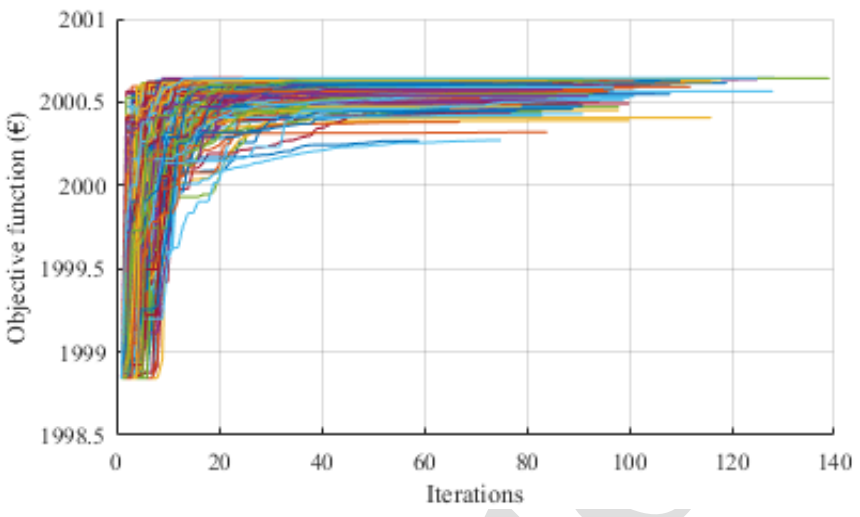

d)

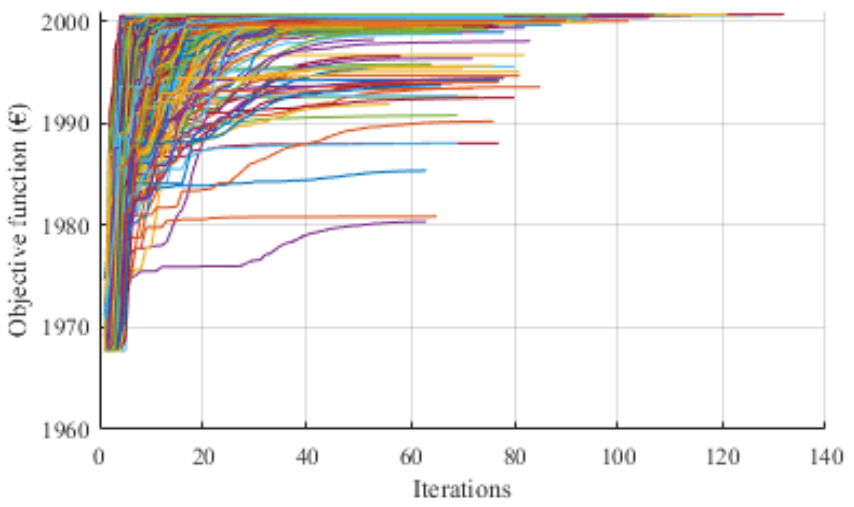

f)

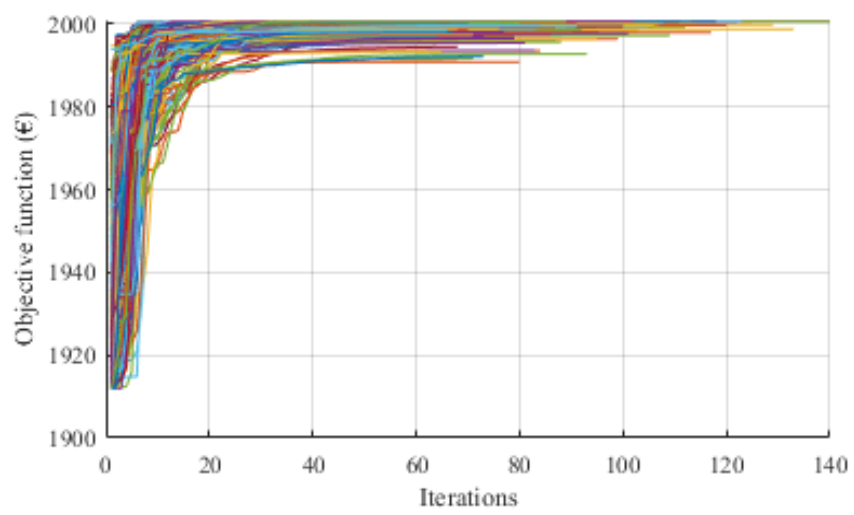

. $\mathrm{STD}=2.5$, d). $\mathrm{STD}=1.5, \mathbf{e}) . \mathrm{STD}=0.5$ and f). $\mathrm{STD}=0.5,(>=0)$

markets are considered. Each bar of each method corresponds to the value of each variable, so have two possible actions for each market that gives a total of ten variables and ten bars in each method. Table 4 shows the values corresponding to the scaling of the result of the method that obtained the maximum objective function value, Cplex 1 , and the results obtained by Cplex2. 


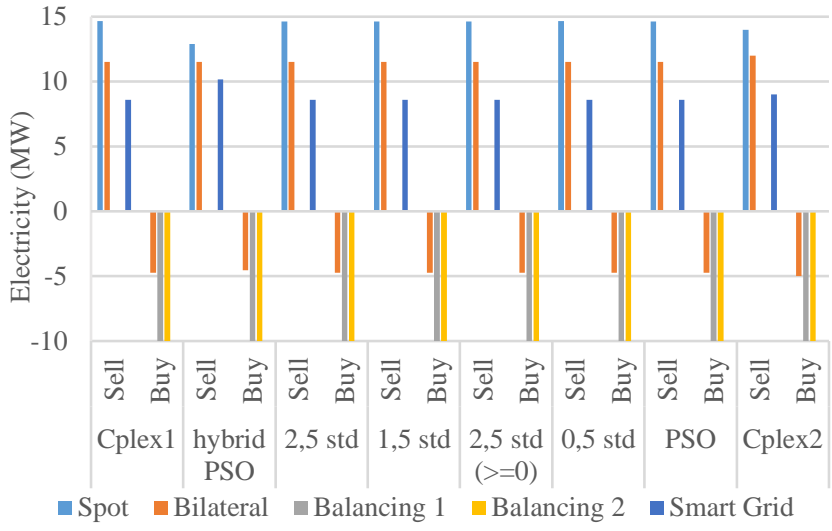

Fig. 5. Sale and purchase in the different markets

Table 4. Scheduling of sale and purchase in the different markets

\begin{tabular}{cccc}
\hline \hline Method & Markets & Sale (MW) & Purchase (MW) \\
\hline \multirow{7}{*}{ Cplex1 } & Spot & 14,64660096 & 0 \\
& Bilateral & 11,5 & 4,729961937 \\
& Balancing 1 & 0 & 10 \\
& Balancing 2 & 0 & 10 \\
& Smart Grid & 8,583360976 & 0 \\
& Spot & 14 & 0 \\
& Bilateral & 12 & 5 \\
Cplex2 & Balancing 1 & 0 & 10 \\
& Balancing 2 & 0 & 10 \\
& Smart Grid & 9 & 0 \\
\hline \hline
\end{tabular}

The representation of Table 4 shows the difference in the deterministic resolutions from the full and simplified versions of the exact resolution method. As one can see, using Cplex2 the variables only contain positive integer values, on the other hand in Cplex1 the variables are numbers with several decimal places. From the result of the objective function of the Table 2, one can see that there is difference in the solutions, and from the analysis of Table 3 the execution time is also different, with about $0.06 \%$ of the time of Cplex 1, Cplex 2 can obtain a solution 0.09\% inferior to Cplex1.

Analyzing the Cplex1 scheduling results, it can be concluded that the method respected the imposed rules that were defined, as it can be observed in the Spot market the sale was not premised and as can see from the Table 4 the variable of this case is 0 , another of the conditions was the fact that in the Balancing markets only one of the actions is allowed and thus it happens, and only the action (buy electricity) is realized, keeping the variable of sales to 0 , in the other two markets the two actions are realized because as the price is variable with the quantity of purchase and can occur multiple opportunities which can resulted in positive profit.

\section{CONCLUSIONS}

This paper presented a novel hybrid optimization model based on the combination between a PSO approach and a simplified resolution using an exact method, to solve the portfolio optimization problem for multiple electricity markets participation. Results enable concluding that the proposed hybrid resolution has advantages in solving the problem, as can be observed by comparing the proposed approach results with those of the standard PSO, in which the algorithm starts the search with a random solution. Using the standard PSO in this problem, a very high STD is obtained; while using the proposed approach the STD decreased. This represents a great advantage, since this measurement gives the indication of the dispersion solution of population around the mean. As can be seen there was also a large increase in the mean objective function value that is achieved, being located near the maximum reference value (Cplex1).

Another of the advantageous conclusions refers to the execution time, because with this method the execution time decreases considerably compared to the time of the reference result using the exact method to solve the complete version of the problem. In this sense, since in electricity markets negotiations, decisions must be taken in short times, this methodology can bring high benefits for real world application.

As future work, intend to expand this methodology by combining different methods such as genetic algorithms and simulated annealing, as well as by using simple metaheuristics to select the initial solution (e.g Vortex Search algorithm (VSA), [21]). In another phase, it is also envisaged to include a risk component in the model and thus obtaining a multiobjective problem so as to be solved with a variation of the proposed methodology.

\section{REFERENCES}

[1] J. Puchinger and R. Raidl, "Combining Metaheuristics and Exact Algorithms in Combinatorial Optimization: A Survey and Classification," Artif. Intell. Knowl. Eng. Appl. a Bioinspired Approach, vol. 3562, pp. 41-53, 2005.

[2] I. H. Osman and G. Laporte, "Metaheuristics: A bibliography," Ann. Oper. Res., vol. 63, no. 5, pp. 511-623, 1996.

[3] L. Meeus and R. Belmans, "Electricity market integration in Europe," Revue-E, vol. 124, no. 1, pp. 5-10, 2008.

[4] European Commission, "Horizon 2020: The EU Framework Programme for Research and Innovation," 2013. [Online]. Available: https://www.rc.auth.gr/Announcement/Details/Item/5837. [Accessed: 17-Jul-2017].

[5] T. H. Yoo, W. Ko, C. H. Rhee, and J. K. Park, "The incentive announcement effect of demand response on market power mitigation in the electricity market," Renew. Sustain. Energy Rev., vol. 76, pp. 545554, 2017.

[6] Z. Vale, T. Pinto, H. Morais, I. Praça, and P. Faria, "VPP's multi-level negotiation in smart grids and competitive electricity markets," IEEE Power and Energy Society General Meeting. pp. 1-8, 2011.

[7] P. Palensky and D. Dietrich, "Demand Side Management: Demand Response, Intelligent Energy Systems, and Smart Loads," IEEE Trans. Ind. Informatics, vol. 7, no. 3, pp. 381-388, 2011.

[8] A. Ciarreta, M. P. Espinosa, and C. Pizarro-Irizar, "Has renewable energy induced competitive behavior in the Spanish electricity market?," Energy Policy, vol. 104, pp. 171-182, 2017.

[9] Z. Vale, T. Pinto, I. Praça, and H. Morais, "MASCEM: Electricity Markets Simulation with Strategic Agents," IEEE Intell. Syst., vol. 26, no. 2, pp. 9-17, Mar. 2011. 
[10] T. Pinto et al., "Adaptive Portfolio Optimization for Multiple Electricity Markets Participation," Neural Networks Learn. Syst. IEEE Trans., vol. PP, no. 99, p. 1, 2015.

[11] H. Markowitz, "Portfolio Selection," J. Finance, vol. 7, no. 1, p. 77, Mar. 1952.

[12] M. Denton, A. Palmer, R. Masiello, and P. Skantze, "Managing market risk in energy," IEEE Trans. Power Syst., vol. 18, no. 2, pp. 494-502, May 2003.

[13] T. Pinto, T. M. Sousa, and Z. Vale, "Dynamic artificial neural network for electricity market prices forecast," Intelligent Engineering Systems (INES), 2012 IEEE 16th International Conference on. pp. 311-316, 2012.

[14] J. Kennedy and R. Eberhart, "Particle swarm optimization," Neural Networks, 1995. Proceedings., IEEE Int. Conf., vol. 4, pp. 1942-1948 vol.4, 1995.

[15] D. Pinheiro Neto, E. G. Domingues, A. P. Coimbra, A. T. de Almeida, A. J. Alves, and W. P. Calixto, "Portfolio optimization of renewable energy assets: Hydro, wind, and photovoltaic energy in the regulated market in Brazil," Energy Econ., vol. 64, pp. 238-250, 2017.
[16] I. Boussaïd, J. Lepagnot, and P. Siarry, "A survey on optimization metaheuristics," Inf. Sci. (Ny)., vol. 237, pp. 82-117, 2013.

[17] M. Mehdi, "Parallel Hybrid Optimization Methods for Permutation Based Problems," no. June, pp. 179-180, 1981.

[18] J. A. Fernández-Vargas, A. Bonilla-Petriciolet, G. P. Rangaiah, and S. E. K. Fateen, "Performance analysis of stopping criteria of populationbased metaheuristics for global optimization in phase equilibrium calculations and modeling," Fluid Phase Equilib., vol. 427, pp. 104-125, 2016.

[19] S. C. Yusta, "Different metaheuristic strategies to solve the feature selection problem," Pattern Recognit. Lett., vol. 30, no. 5, pp. 525-534, 2009.

[20] T. Pinto, Z. Vale, T. M. Sousa, T. Sousa, H. Morais, and I. Praça, "Particle Swarm Optimization of Electricity Market Negotiating Players Portfolio," Highlights Pract. Appl. Heterog. Multi-Agent Syst., vol. 430, pp. 273-284, 2014.

[21] B. Dogan and T. Omez, "A new metaheuristic for numerical function optimization: Vortex Search algorithm," Inf. Sci. (Ny)., vol. 293, pp. $125-145,2015$. 